\title{
Combined oxidative phosphorylation defect type 4
}

INSERM

\section{Source}

INSERM. (1999). Orphanet: an online rare disease and orphan drug data base. Combined oxidative phosphorylation defect type 4. ORPHA:254925

Combined oxidative phosphorylation defect type 4 is a rare mitochondrial disorder due to a defect in mitochondrial protein synthesis characterized by a neonatal onset of severe metabolic acidosis and respiratory distress, persistent lactic acidosis with episodes of metabolic crises, developmental regression, microcephaly, abnormal gaze fixation and pursuit, axial hypotonia with limb spasticity and reduced spontaneous movements. Neuroimaging studies reveal polymicrogyria, white matter abnormalities and multiple cystic brain lesions, including basal ganglia, and cerebral atrophy. Decreased activity of complex I and IV have been determined in muscle biopsy. 